\title{
Cooperative Relay in Cognitive Radio Networks: Decode-and-Forward or Amplify-and-Forward?
}

\author{
Donglin $\mathrm{Hu}$ and Shiwen Mao \\ Dept. ECE, Auburn University, Auburn, AL 36849-5201
}

\begin{abstract}
Cognitive radios (CR) and cooperative communications represent new paradigms that both can effectively improve the spectrum efficiency of future wireless networks. In this paper, we investigate the problem of cooperative relay in CR networks for further improved network performance. The objective is to provide an analysis for the comparison of two representative cooperative relay strategies, decode and forward (DF) and amplify and forward (AF), in the context of CR networks. We consider optimal spectrum sensing and $p$-Persistent CSMA for spectrum access, and derive closed-form expressions for network-wide throughput achieved by DF and AF. Our analysis is validated by simulations. We find each of the strategies performs better in a certain parameter range; there is no case of dominance for the two strategies. The considerable gaps between the cooperative relay results and the direct link results exemplify the diversity gain achieved by cooperative relays in CR networks.
\end{abstract}

\section{INTRODUCTION}

According to Cisco's recent study, wireless data traffic is expected to increase by a factor of 66 times by 2013. Much of this future wireless data traffic will be video based services driven by the need for ubiquitous access to wireless multimedia content. Such drastic increase in traffic demand will significantly stress the capacity of future wireless networks.

Cognitive radios (CR) provide an effective solution to meeting this critical demand, by exploiting co-deployed networks and aggregating underutilized spectrum for future wireless networks [1], [2]. CR was motivated by the spectrum measurements by the FCC, where a significant amount of the assigned spectrum is found to remain underutilized. CR represents a paradigm change in spectrum regulation and access, from exclusive use by primary users to shared spectrum for secondary users, which can enhance spectrum utilization and achieve high throughput capacity. Cooperative communications represents another new paradigm for wireless communications [3], [4]. It allows wireless nodes assisting each other in information delivery, with the objective of gaining greater reliability and efficiency than they could obtain individually (i.e., to achieve the so-called cooperative diversity). It enables opportunistic use of network energy and bandwidth, and delivers salient advantages over legacy point-to-point communications.

Recently, there has been some interesting work on cooperative relay in $\mathrm{CR}$ networks [5], [6]. In [5], the authors considered the case of two single-user links, one primary and one secondary. The secondary transmitter is allowed to act as a "transparent" relay for the primary link, motivated by the rationale that helping primary users will lead to more transmission opportunities for CR nodes. In [6], the authors presented an excellent overview of several cooperative relay scenarios and various related issues. A new MAC protocol was proposed and implemented in a testbed to select a spectrumrich $\mathrm{CR}$ node as relay for a $\mathrm{CR}$ transmitter/receiver pair.

In this paper, we investigate the problem of cooperative relay in CR networks. We assume a primary network with multiple licensed bands and a CR network consisting of multiple cooperative relay links. Each cooperative relay link consists of a $\mathrm{CR}$ transmitter, a $\mathrm{CR}$ relay, and a $\mathrm{CR}$ receiver. The objective is to provide an analysis for the comparison of two representative cooperative relay strategies, i.e., decode and forward (DF) and amplify and forward (AF), in the context of CR networks. We first consider cooperative spectrum sensing by the CR nodes. We model both types of sensing errors, i.e., miss detection and false alarm, and derive the optimal value for the sensing threshold. Next, we incorporate DF and AF into the $p$-Persistent Carrier Sense Multiple Access (CSMA) protocol for channel access for the CR nodes. We develop closed-form expressions for the network-wide capacities achieved by DF and $\mathrm{AF}$, respectively, as well as that for the case of direct link transmission for comparison purpose.

Through analytical and simulation evaluations of DF and AF-based cooperative relay strategies, we find the analysis provides upper bounds for the simulated results, which are reasonably tight. We also find cross-point with the $\mathrm{AF}$ and DF curves when some system parameter is varied, indicating that each of them performs better in a certain parameter range. There is no case that one completely dominates the other for the two strategies. The considerable gaps between the cooperative relay results and the direct link results exemplify the diversity gain achieved by cooperative relays in CR networks.

The remainder of this paper is organized as follows. The system model is described in section II. We analyze the two CR cooperative relay strategies in Section III. Our simulation evaluations are presented in Section IV. Related work is discussed in Section V and Section VI concludes the paper.

\section{SySTEM MODEL}

We assume a primary network and a spectrum band that is divided into $M$ licensed channels, each modeled as a time slotted, block-fading channel. The state of each channel evolves independently following a discrete time Markov process [1]. The status of channel $m$ in time slot $t$ is denoted as $S_{m}(t)$, for $m=1,2, \cdots, M$. When $S_{m}(t)=0$, the channel is in the idle state; when $S_{m}(t)=1$, the channel is in the busy state (i.e., being used by primary users). Let $\lambda_{m}$ and $\mu_{m}$ be the transition probability to remain in state 0 and the transition probability from state 1 to 0 for channel $m$, respectively. The utilization of channel $m$ with respect to primary user transmissions, denoted by $\eta_{m}=\operatorname{Pr}\left\{S_{m}(t)=1\right\}$, can be written as: $\eta_{m}=\lim _{T \rightarrow \infty} \frac{1}{T} \sum_{t=1}^{T} S_{m}(t)=\frac{1-\lambda_{m}}{1-\lambda_{m}+\mu_{m}}$. 
There is a CR network colocated with the primary network. The CR network consists of $N$ sets of cooperative relay links, each including a CR transmitter, a CR relay and a CR receiver. Each CR node (or, secondary user) is equipped with two transceivers, each incorporating a software defined radio (SDR) that is able to tune to any of the $M$ licensed channels and a control channel and operate from there.

We assume CR nodes access the licensed channels following the same time slot structure [1]. Each time slot is divided into two phases, the sensing phase and the transmission phase. In the sensing phase, a CR node chooses one of the $M$ channels to sense using one of its transceivers, and then exchanges sensed channel information with other CR nodes using the other transceiver over the control channel. During the transmission phase, the CR transmitter and/or relay transmit data frames on licensed channels that are believed to be idle based on sensing results, using one or both of the transceivers. We consider cooperative relay strategies $\mathrm{AF}$ and $\mathrm{DF}$, and compare their performance in the following sections.

\section{Cooperative Relay IN CR Networks}

In this section, we present an analysis of the cooperative relay strategies in CR networks. We first examine cooperative spectrum sensing and derive the optimal sensing threshold. We then consider cooperative relay and spectrum access, and derive the network-wide throughput performance.

\section{A. Spectrum Sensing}

Although precise and timely channel state information is desirable for spectrum access and primary user protection, continuous, full-spectrum sensing is both energy inefficient and hardware demanding [1]. Since each CR node is equipped with two transceivers and one has to be used for distributing sensing results over the control channel, it can sense only one of the channels at a time, using the remaining transceiver. We assume that each CR node senses a fixed channel throughout the time slots and receives from the control channel sensing results on other channels, i.e., distributed by other CR nodes.

During the sensing process, two kinds of detection errors may occur. With a false alarm, an idle channel is considered busy and a spectrum opportunity will be wasted. With a miss detection, a busy channel is considered idle, which may lead to collision with primary users. We adopt hypothesis test to detect the availability of channel $\mathrm{m}$. The null hypothesis $H_{0}^{m}$ is "channel $m$ is idle." The alternative hypothesis $H_{1}^{m}$ is "channel $m$ is busy." Let $\epsilon_{i}^{m}$ and $\delta_{i}^{m}$ be the probabilities of false alarm and miss detection, respectively, when CR node $i$ senses channel $m$. We have $\epsilon_{i}^{m}=\operatorname{Pr}\left\{\Theta_{i}^{m}=1 \mid H_{0}^{m}\right\}$ and $\delta_{i}^{m}=\operatorname{Pr}\left\{\Theta_{i}^{m}=0 \mid H_{1}^{m}\right\}$, where $\Theta_{i}^{m} \in\{0,1\}$ is the channel $m$ sensing result at node $i$.

Assume there are $N_{m} \mathrm{CR}$ nodes sensing channel $m$. After the sensing phase, each CR node obtains a sensing result vector $\vec{\Theta}_{m}=\left[\Theta_{1}^{m}, \Theta_{2}^{m}, \cdots, \Theta_{N_{m}}^{m}\right]$ for channel $m$. The conditional probability $a_{m}\left(\vec{\Theta}_{m}\right)$ on channel $m$ availability is

$$
\begin{aligned}
& a_{m}\left(\Theta_{1}^{m}, \Theta_{2}^{m}, \cdots, \Theta_{N_{m}}^{m}\right) \cong \operatorname{Pr}\left\{H_{0}^{m} \mid \Theta_{1}^{m}, \Theta_{2}^{m}, \cdots, \Theta_{N_{m}}^{m}\right\} \\
& =\frac{\left.\operatorname{Pr}\left\{\Theta_{1}^{m}, \Theta_{2}^{m}, \cdots, \Theta_{N_{m}}^{m} \mid H_{0}^{m}\right)\right\} \operatorname{Pr}\left\{H_{0}^{m}\right\}}{\sum_{j \in\{0,1\}} \operatorname{Pr}\left\{\Theta_{1}^{m}, \Theta_{2}^{m}, \cdots, \Theta_{N_{m}}^{m} \mid H_{j}^{m}\right\} \operatorname{Pr}\left\{H_{j}^{m}\right\}}
\end{aligned}
$$

$$
\begin{aligned}
& =\frac{\prod_{i=1}^{N_{m}} \operatorname{Pr}\left\{\Theta_{i}^{m} \mid H_{0}^{m}\right\} \operatorname{Pr}\left\{H_{0}^{m}\right\}}{\sum_{j \in\{0,1\}} \prod_{i=1}^{N_{m}} \operatorname{Pr}\left\{\Theta_{i}^{m} \mid H_{j}^{m}\right\} \operatorname{Pr}\left\{H_{j}^{m}\right\}} \\
& =\left[1+\frac{\operatorname{Pr}\left\{H_{1}^{m}\right\}}{\operatorname{Pr}\left\{H_{0}^{m}\right\}} \prod_{i=1}^{N_{m}} \frac{\operatorname{Pr}\left\{\Theta_{i}^{m} \mid H_{1}^{m}\right\}}{\operatorname{Pr}\left\{\Theta_{i}^{m} \mid H_{0}^{m}\right\}}\right]^{-1} \\
& =\left[1+\frac{\eta_{m}}{1-\eta_{m}} \prod_{i=1}^{N_{m}} \frac{\left(\delta_{i}^{m}\right)^{1-\Theta_{i}^{m}}\left(1-\delta_{i}^{m}\right)^{\Theta_{i}^{m}}}{\left(\epsilon_{i}^{m}\right)^{\Theta_{i}^{m}}\left(1-\epsilon_{i}^{m}\right)^{1-\Theta_{i}^{m}}}\right]^{-1} .
\end{aligned}
$$

If $a_{m}\left(\vec{\Theta}_{m}\right)$ is greater than a sensing threshold $\tau_{m}$, channel $m$ is believed to be idle; otherwise, channel $m$ is believed to be busy. The decision variable $D_{m}$ is defined as follows.

$$
D_{m}= \begin{cases}0, & \text { if } a_{m}\left(\vec{\Theta}_{m}\right)>\tau_{m} \\ 1, & \text { if } a_{m}\left(\vec{\Theta}_{m}\right) \leq \tau_{m} .\end{cases}
$$

CR nodes only attempt to access channel $m$ where $D_{m}$ is 0 . Since function $a_{m}\left(\vec{\Theta}_{m}\right)$ in (1) has $N_{m}$ binary variables, there can be $2^{N_{m}}$ different combinations corresponding to $2^{N_{m}}$ values for $a_{m}\left(\vec{\Theta}_{m}\right)$. We sort the $2^{N_{m}}$ combinations according to their $a_{m}\left(\vec{\Theta}_{m}\right)$ values in the non-increasing order. Let $a_{m}^{(j)}$ be the $j$ th largest function value and $\vec{\theta}_{m}^{(j)}$ the argument that achieves the $j$ th largest function value $a_{m}^{(j)}$, where $\vec{\theta}_{m}^{(j)}=$ $\left[\theta_{1}^{m}(j), \theta_{2}^{m}(j), \cdots, \theta_{N_{m}}^{m}(j)\right]$.

In the design of CR networks, we consider two objectives: (i) how to avoid harmful interference to primary users, and (ii) how to fully exploit spectrum opportunities for the CR nodes. For primary user protection, we limit the collision probability with primary user with a threshold. Let $\gamma_{m}$ be the tolerance threshold, i.e., the maximum allowable interference probability with primary users on channel $m$. The probability of collision with primary users on channel $m$ is given as $\operatorname{Pr}\left\{D_{m}=0 \mid H_{1}^{m}\right\}$; the probability of detecting an available transmission opportunity is $\operatorname{Pr}\left\{D_{m}=0 \mid H_{0}^{m}\right\}$. Our objective is to maximize the probability of detecting available channels, while keeping the collision probability below $\gamma_{m}$, i.e.,

$$
\begin{aligned}
\max _{\tau_{m}} & \operatorname{Pr}\left\{D_{m}=0 \mid H_{0}^{m}\right\} \\
\text { subect to: } & \operatorname{Pr}\left\{D_{m}=0 \mid H_{1}^{m}\right\} \leq \gamma_{m} .
\end{aligned}
$$

From their definitions, both $\operatorname{Pr}\left\{D_{m}=0 \mid H_{1}^{m}\right\}$ and $\operatorname{Pr}\left\{D_{m}=0 \mid H_{0}^{m}\right\}$ are decreasing functions of $\tau_{m}$. As $\left.\operatorname{Pr}\left\{D_{m}=0 \mid H_{1}^{m}\right)\right\}$ approaches its maximum allowed value $\gamma_{m}, \operatorname{Pr}\left\{D_{m}=0 \mid H_{0}^{m}\right\}$ also approaches its maximum. Therefore, solving the optimization problem (3) $\sim(4)$ is equivalent to solving $\operatorname{Pr}\left\{D_{m}=0 \mid H_{1}^{m}\right\}=\gamma_{m}$. If $\tau_{m}=a_{m}^{(j)}$, we have

$$
\begin{aligned}
& \operatorname{Pr}\left\{D_{m}=0 \mid H_{1}^{m}\right\}\left(a_{m}^{(j)}\right)=\operatorname{Pr}\left\{a_{m}\left(\vec{\Theta}_{m}\right)>a_{m}^{(j)} \mid H_{1}^{m}\right\} \\
= & \sum_{l=1}^{j-1} \operatorname{Pr}\left\{a_{m}\left(\vec{\Theta}_{m}\right)=a_{m}^{(l)} \mid H_{1}^{m}\right\}=\sum_{l=1}^{j-1}\left(\delta_{i}^{m}\right)^{1-\theta_{i}^{m}(l)}\left(1-\delta_{i}^{m}\right)^{\theta_{i}^{m}(l)}
\end{aligned}
$$

Obviously, $\operatorname{Pr}\left\{D_{m}=0 \mid H_{1}^{m}\right\}\left(a_{m}^{(j)}\right)$ is an increasing function of $j$. The optimal sensing threshold $\tau_{m}^{*}$ can be set to $a_{m}^{(j)}$, such that $\operatorname{Pr}\left\{D_{m}=0 \mid H_{1}^{m}\right\}\left(a_{m}^{(j)}\right) \leq \gamma_{m}$ and $\operatorname{Pr}\left\{D_{m}=\right.$ $\left.0 \mid H_{1}^{m}\right\}\left(a_{m}^{(j+1)}\right)>\gamma_{m}$. The algorithm for computing the optimal sensing threshold $\tau_{m}^{*}$ is presented in Table I. 
TABLE I

Algorithm For COMPuting THE Optimal SENSing THRESHOLD

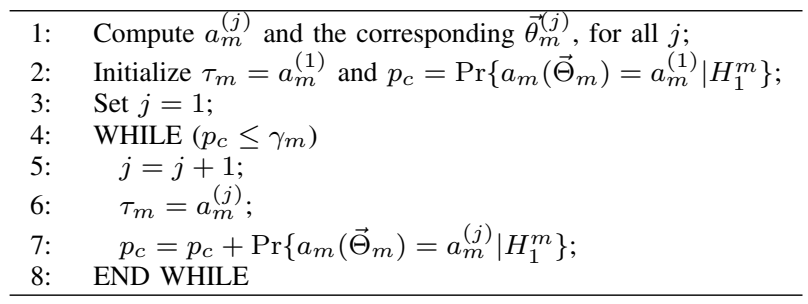

Once the optimal sensing threshold $\tau_{m}^{*}$ is determined, $\operatorname{Pr}\left\{D_{m}=0 \mid H_{1}^{m}\right\}$ can be computed as given in (5) and $\operatorname{Pr}\left\{D_{m}=0 \mid H_{0}^{m}\right\}$ can be computed as:

$$
\begin{aligned}
& \operatorname{Pr}\left\{D_{m}=0 \mid H_{0}^{m}\right\}=\operatorname{Pr}\left\{a_{m}\left(\vec{\Theta}_{m}\right)>\tau_{m}^{*} \mid H_{0}^{m}\right\} \\
= & \sum_{l=1}^{j-1} \operatorname{Pr}\left\{a_{m}\left(\vec{\Theta}_{m}\right)=a_{m}^{(l)} \mid H_{0}^{m}\right\}=\sum_{l=1}^{j-1}\left(\epsilon_{i}^{m}\right)^{\theta_{i}^{m}(l)}\left(1-\epsilon_{i}^{m}\right)^{1-\theta_{i}^{m}(l)}
\end{aligned}
$$

\section{B. Cooperative Relay}

During the transmission phase, CR transmitters and relays attempt to send data through the channels that are believed to be idle. We assume fixed length data frames. Let $G_{1}^{k}$ and $G_{2}^{k}$ denote the path gains from the transmitter to relay and from the relay to receiver, respectively, and let $\sigma_{r, k}^{2}$ and $\sigma_{d, k}^{2}$ denote the noise powers at the relay and receiver, respectively, for the $k$ th cooperative relay link. We examine the two cooperation relay strategies DF and AF, and direct link transmission below.

1) Decode-and-Forward: With DF, the CR transmitter sends data frames to its relay in an odd time slot. The relay decodes the data and forwards it to the receiver in the following even time slot. The receiver can successfully decode the frame if it is not lost or corrupted on both links. We assume a data frame can be successfully decoded if the received signalto-noise ratio (SNR) is no less than a decoding threshold $\kappa$. We assume gains on different links are independent to each other. The decoding rate of DF at the $k$ th receiver, denoted by $P_{D F}^{k}$, can be computed as,

$$
\begin{aligned}
P_{D F}^{k} & =\operatorname{Pr}\left\{\left(P_{s} G_{1}^{k} / \sigma_{r, k}^{2} \geq \kappa\right) \text { and }\left(P_{r} G_{2}^{k} / \sigma_{d, k}^{2} \geq \kappa\right)\right\} \\
& =\bar{F}_{G_{1}^{k}}\left(\sigma_{r, k}^{2} \kappa / P_{s}\right) \bar{F}_{G_{2}^{k}}\left(\sigma_{d, k}^{2} \kappa / P_{r}\right)
\end{aligned}
$$

where $P_{s}$ and $P_{r}$ are the transmit powers at the transmitter and relay, respectively, $\bar{F}_{G_{1}^{k}}(x)$ and $\bar{F}_{G_{2}^{k}}(x)$ are the complementary cumulative distribution functions (CCDF) of path gains $G_{1}^{k}$ and $G_{2}^{k}$, respectively.

2) Amplify-and-Forward: With AF, the relay amplifies the received signal and forwards it to the receiver in the same time slot. The relay receives data from the transmitter using one transceiver operating on one or more idle channels; it forwards the data simultaneously to the receiver using the other transceiver operating on one or more different idle channels. A data frame can be successfully decoded if the SNR at the receiver is no less than $\kappa$. The decoding rate of AF at the $k$ th receiver, denoted as $P_{A F}^{k}$, can be computed as,

$$
\begin{aligned}
P_{A F}^{k} & =\operatorname{Pr}\left\{\frac{P_{r}}{G_{1}^{k} P_{s}+\sigma_{r, k}^{2}} \frac{P_{s} G_{1}^{k} G_{2}^{k}}{\sigma_{d, k}^{2}} \geq \kappa\right\} \\
& =\int_{0}^{+\infty} \bar{F}_{G_{2}^{k}}\left(\frac{\left(P_{s} x+\sigma_{r, k}^{2}\right) \sigma_{d, k}^{2} \kappa}{P_{s} P_{r} x}\right) d F_{G_{1}^{k}}(x) .
\end{aligned}
$$

3) Direct Link Transmission: For comparison purpose, we also consider the case of direct link transmission (DL), where the CR transmitter transmits to the receiver via the direct link without using the relay. Let the path gain be $G_{0}^{k}$ with CCDF $\bar{F}_{G_{0}^{k}}(x)$ for the $k$ th direct link. Following similar analysis, the decoding rate of DL at the $k$ th receiver, denoted as $P_{D L}^{k}$, can be computed as,

$$
P_{D L}^{k}=\operatorname{Pr}\left\{P_{s} G_{0}^{k} / \sigma_{d, k}^{2} \geq \kappa\right\}=\bar{F}_{G_{0}^{k}}\left(\sigma_{d, k}^{2} \kappa / P_{s}\right) .
$$

\section{Channel Access}

We assume greedy transmitters that always have data to send. The CR nodes use $p$-Persistent CSMA for channel access. At the beginning of the transmission phase of an odd time slot, CR transmitters send Request-to-Send (RTS) with probability $p$ over the control channel. Since there are $N \mathrm{CR}$ transmitters, the transmission probability $p$ is set to $1 / N$ to maximize the throughput (i.e., to maximize $P_{1}$ in (10) given below). Three cases may occur:

- no CR transmitter sends RTS for channel access.

- only one CR transmitter sends RTS, and it successfully receives Clear-to-Send (CTS) from the receiver over the control channel. It then accesses some of or all the channels that are believed to be idle for data transmission.

- more than one CR transmitters send RTS and collision occurs on the control channel. No CR node can access the licensed channels.

Let $P_{0}, P_{1}$ and $P_{2}$ denote the probability corresponding to the three cases enumerated above, respectively. We have

$$
\left\{\begin{array}{l}
P_{0}=(1-p)^{N}=(1-1 / N)^{N} \\
P_{1}=N p(1-p)^{N-1}=(1-1 / N)^{N-1} \\
P_{2}=1-P_{0}-P_{1} .
\end{array}\right.
$$

The CR cooperative relay link that wins the channels in the odd time slot will continue to use the channels in the following even time slot. A new round of channel competition will start in the next odd time slot following these two time slots.

Since a licensed channel is accessed with probability $P_{1}$ in the odd time slot, we modify the tolerance threshold $\gamma_{m}$ as $\gamma_{m}^{\prime}=\gamma_{m} / P_{1}$, such that the maximum allowable collision requirement can still be satisfied. In the even time slot, the channels will continue to be used by the winning cooperative relay link, i.e., to be accessed with probability 1 . Therefore the tolerance threshold is still $\gamma_{m}$ for even time slots.

\section{Capacity Analysis}

Once the CR transmitter wins the competition, as indicated by received CTS, it begins to send data over the licensed channels that are inferred to be idle (i.e., $D_{m}=0$ ). We assume the channel bonding and aggregation technique is used, such that multiple channels can be used collectively by a CR node for data transmission [7]. 
With DF, the winning CR transmitter uses all the available channels to transmit to the relay in the odd time slot. In the following even time slot, the CR transmitter stops transmission, while the relay uses the available channels in the even time slot to forward data to the receiver. If the number of available channels in the even time slot is equal to or greater than that in the odd time slot, the relay uses the same number of channels to forward all the received data. Otherwise, the relay uses all the available channels to forward part of the received data; the excess data will be dropped due to limited channel resource in the even time slot. The dropped data will be retransmitted in some future odd time slot by the transmitter.

With AF, no matter it is an odd or even time slot, the CR transmitter always uses half of the available licensed channels to transmit to the relay. The relay uses one of its transceivers to receive from the chosen half of the available channels. Simultaneously, it uses the other transceiver to forward the received data to the receiver using the remaining half of the available channels. Let $D_{m}^{o d}$ and $D_{m}^{e v}$ be the decision variables of channel $m$ in the odd and even time slot, respectively (see (2)). Let $S_{m}^{o d}$ and $S_{m}^{e v}$ be the status of channel $m$ in the odd and even time slot, respectively. We have,

$$
\begin{aligned}
& \operatorname{Pr}\left\{D_{m}^{o d}=i, S_{m}^{o d}=j, D_{m}^{e v}=k, S_{m}^{e v}=l\right\} \\
= & \operatorname{Pr}\left\{D_{m}^{e v}=k \mid S_{m}^{e v}=l\right\} \operatorname{Pr}\left\{D_{m}^{o d}=i \mid S_{m}^{o d}=j\right\} \times \\
& \operatorname{Pr}\left\{S_{m}^{e v}=l \mid S_{m}^{\text {od }}=j\right\} \operatorname{Pr}\left\{S_{m}^{o d}=j\right\}, \text { for } i, j, k, l \in\{0,1\} .
\end{aligned}
$$

where $\operatorname{Pr}\left\{S_{m}^{o d}=j\right\}$ are the probabilities that channel $m$ is busy or idle, $\operatorname{Pr}\left\{S_{m}^{e v}=l \mid S_{m}^{o d}=j\right\}$ are the channel $m$ transition probabilities. $\operatorname{Pr}\left\{D_{m}^{e v}=k \mid S_{m}^{e v}=l\right\}$ and $\operatorname{Pr}\left\{D_{m}^{o d}=i \mid S_{m}^{o d}=j\right\}$ can be computed as in (5) and (6).

Let $N_{D F}, N_{A F}$ and $N_{D L}$ be the number of frames successfully delivered to the receiver in the two consecutive time slots using DF, AF and DL, respectively. Define $\bar{S}_{m}^{o d}=1-S_{m}^{o d}$, $\bar{S}_{m}^{e v}=1-S_{m}^{e v}, \bar{D}_{m}^{o d}=1-D_{m}^{o d}$ and $\bar{D}_{m}^{e v}=1-D_{m}^{e v}$. We have

$$
\left\{\begin{array}{l}
N_{D F}=\left(\sum_{m=1}^{M} \bar{S}_{m}^{o d} \bar{D}_{m}^{o d}\right) \wedge\left(\sum_{m=1}^{M} \bar{S}_{m}^{e v} \bar{D}_{m}^{e v}\right) \\
N_{A F}=\left\lfloor\frac{1}{2} \sum_{m=1}^{M} \bar{S}_{m}^{o d} \bar{D}_{m}^{o d}\right\rfloor+\left\lfloor\frac{1}{2} \sum_{m=1}^{M} \bar{S}_{m}^{e v} \bar{D}_{m}^{e v}\right\rfloor \\
N_{D L}=\left(\sum_{m=1}^{M} \bar{S}_{m}^{o d} \bar{D}_{m}^{o d}\right)+\left(\sum_{m=1}^{M} \bar{S}_{m}^{e v} \bar{D}_{m}^{e v}\right),
\end{array}\right.
$$

where $x \wedge y$ represents the minimum of $x$ and $y$, and $\lfloor x\rfloor$ means the maximum integer that is not larger than $x$.

As discussed, the probability that a frame can be successfully delivered is $P_{D F}^{k}, P_{A F}^{k}$, or $P_{D L}^{k}$ for the three schemes, respectively. Recall that time slot/channels are allocated distributedly every two time slots. We derive the capacity for the three schemes as

$$
\left\{\begin{array}{l}
C_{D F}=\mathrm{E}\left[N_{D F}\right] \cdot \sum_{k=1}^{N}\left(P_{D F}^{k} P_{1} L\right) /\left(2 N T_{s}\right) \\
C_{A F}=\mathrm{E}\left[N_{A F}\right] \cdot \sum_{k=1}^{N}\left(P_{A F}^{k} P_{1} L\right) /\left(2 N T_{s}\right) \\
C_{D L}=\mathrm{E}\left[N_{D L}\right] \cdot \sum_{k=1}^{N}\left(P_{D L}^{k} P_{1} L\right) /\left(2 N T_{s}\right),
\end{array}\right.
$$

where $L$ is the packet length and $T_{s}$ is the duration of a time slot. The expectations are computed using (11) (12).

\section{Performance Evaluation}

We evaluate the performance of the cooperative relay strategies with analysis and simulations. The analytical capacities of
TABLE II

SIMULATION PARAMETERS

\begin{tabular}{lll}
\hline Symbol & Value & Definition \\
\hline$M$ & 5 & number of licensed channels \\
$\lambda$ & 0.7 & channel transition probability from idle to idle \\
$\mu$ & 0.2 & channel transition probability from busy to idle \\
$\eta$ & 0.6 & channel utilization \\
$\gamma$ & 0.08 & maximum allowable collision probability \\
$N$ & 7 & number of CR cooperative relay links \\
$P_{s}$ & $10 \mathrm{dBm}$ & transmit power of the CR transmitters \\
$P_{r}$ & $10 \mathrm{dBm}$ & transmit power of CR relays \\
$L$ & $1 \mathrm{~kb}$ & packet length \\
$T_{s}$ & $1 \mathrm{~ms}$ & duration of a time slot \\
\hline
\end{tabular}

the schemes are obtained as in Section III. The actual throughput is obtained using MATLAB simulations. The simulation parameters are listed in Table II, unless specified otherwise. We consider five licensed channels and a CR network with seven cooperative relay links. The channels have identical parameters for the Markov chain models. Each point in the simulation curves is the average of 10 simulation runs with different random seeds. We plot $95 \%$ confidence intervals for the simulation results, which are negligible in all the cases.

We first examine the impact of the number of licensed channels. To illustrate the effect of spectrum sensing, we let the decoding rate $P_{A F}^{k}$ be equal to $P_{D F}^{k}$. In Fig. 1(a), we plot the throughput of $\mathrm{AF}, \mathrm{DF}$, and DL under increased number of licensed channels. The analytical curves are upper bounds for the simulation curves in all the cases, and the gap between the two is reasonably small. Furthermore, as the number of license channels is increased, the throughput of both AF and $\mathrm{DF}$ are increased. The slope of the AF curves is larger than that of the DF curves. There is a cross point between five and six, as predicted by both simulation and analysis curves. This indicates that AF outperforms DF when the number of channels is large. This is because AF is more flexible than DF in exploiting the idle channels in the two consecutive time slots. The DL analysis and simulation curves also increases with the number of channels, but with the lowest slope and the lowest throughput values.

In Fig. 1(b), we demonstrate the impact of channel utilization on the throughput of the schemes. The channel utilization $\eta$ is increased from 0.3 to 0.9 , when primary users get more active. As $\eta$ is increased, the transmission opportunities for CR nodes are reduced and all the throughputs are degraded. We find the throughputs of AF and DF are close to each other when the channel utilization is high. AF outperforms DF in the low channel utilization region, but is inferior to DF in the high channel utilization region. There is a cross point between the $\mathrm{AF}$ and $\mathrm{DF}$ curves between $\eta=0.5$ and $\eta=0.6$. When the channel utilization is low, there is a big gap between the cooperative relay curves and and the DL curves.

In Fig. 1(c), we examine the channel fading factor. We consider Rayleigh block fading channels, where the received power is exponentially distributed with a distance-dependent mean. We fix the transmitter power at $10 \mathrm{dBm}$, and increase the relay power from one $\mathrm{dBm}$ to $18 \mathrm{dBm}$. As the relay power is increased, the throughput is also increased since the SNR at the receiver is improved. We can see the increasing speed of $\mathrm{AF}$ is larger than that of $\mathrm{DF}$, indicating that $\mathrm{AF}$ has superior performance than DF when the relay transmit power is large. The capacity analysis also demonstrate the same trend. The 


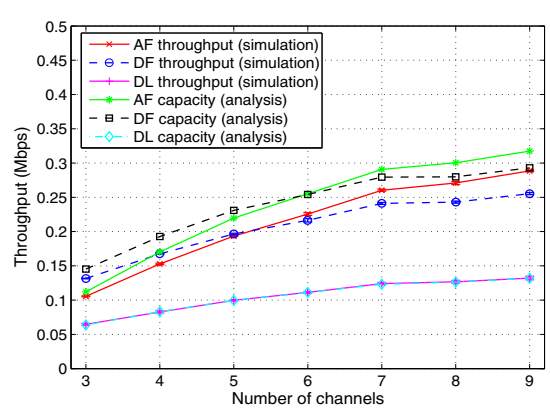

(a) Number of licensed channels

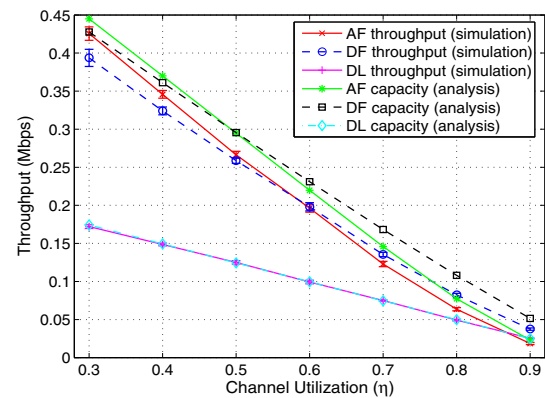

(b) Primary user channel utilization

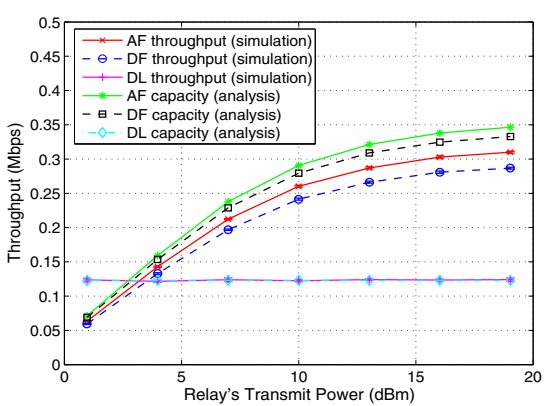

(c) Transmit power of relay nodes

Fig. 1. Throughput performance versus system parameters.

throughput of DL does not depend on the relay node. Its throughput is better than that of $\mathrm{AF}$ and $\mathrm{DF}$ when the relay transmit power is low, since both $\mathrm{AF}$ and $\mathrm{DF}$ are limited by the relay-to-receiver link in this low power region. However, the throughputs of AF and DF quickly exceed that of DL and grow fast as the relay-to-receiver link is improved with the increased relay transmit power. The considerable gaps between the cooperative relay link curves and the DL curves in Figs. 1(a), 1(b) and 1(c) exemplify the diversity gain achieved by cooperative relays in $\mathrm{CR}$ networks.

\section{RELATED WORK}

The theoretical foundation of relay channels was laid by the seminal work [8]. The capacities of the Gaussian relay channel and certain discrete relay channels are evaluated, and the achievable lower bound to the capacity of the general relay channel is established in this work. In [3], the authors described cooperative diversity, where diversity gains are achieved via the cooperation of mobile users. In [4], the authors developed and analyzed low-complexity cooperative diversity protocols. Several cooperative strategies, including $\mathrm{AF}$ and $\mathrm{DF}$, were described and their performance characterizations were derived in terms of outage probabilities.

In practice, there is a restriction that each node cannot transmit and receive simultaneously in the same frequency band. The "cheap" relay channel concept was introduced in [9], where the authors derived the capacity of the Gaussian degraded "cheap" relay channel. Multiple relay nodes for a transmitter-receiver pair are investigated in [10] and [11]. The authors showed that, when compared with complex protocols that involve all relays, the simplified protocol with no more than one relay chosen can achieve the same performance. This is the reason why we consider single relay in this paper.

In [12], Ng and Yu proposed a utility maximization framework for joint optimization of node, relay strategy selection, and power, bandwidth and rate allocation in a cellular network. Cai et al. [13] presented a semi-distributed algorithm for $\mathrm{AF}$ relay networks. A heuristic was adopted to select relay and allocate power. Both AF and DF were considered in [14], where a polynomial time algorithm for optimal relay selection was developed and proved to be optimal. In [15], a protocol is proposed for joint routing, relay selection, and dynamic spectrum allocation for multi-hop CR networks, and its performance is evaluated through simulations.

\section{CONCLUSION}

In this paper, we studied the problem of cooperative relay in CR networks. We modeled the two cooperative relay strategies, i.e., DF and AF, which are integrated with $p$-Persistent CSMA. We analyzed their throughput performance and compared them under various parameter ranges. Cross-point with the AF and DF curves are found when some parameter is varied, indicating that each of them performs better in a certain parameter range; there is no case of dominance for the two strategies.

\section{ACKNOWLEDGMENT}

This work is supported in part by the NSF under Grants ECCS-0802113, IIP-0738088 and IIP-1032002.

\section{REFERENCES}

[1] Q. Zhao and B. Sadler, "A survey of dynamic spectrum access," IEEE Signal Process., vol.24, no.3, pp.79-89, May 2007.

[2] Y. Zhao, S. Mao, J. Neel, and J. H. Reed, "Performance evaluation of cognitive radios: Metrics, utility functions, and methodologies," Proc. IEEE, vol. 97, no. 4, pp. 642-659, Apr. 2009.

[3] A. Sendonaris, E. Erkip, and B. Aazhang, "User cooperation diversity. part I. system description," IEEE Trans. Commun., vol. 51, no. 11, pp. 1927-1938, Nov. 2003.

[4] N. Laneman, D. Tse, and G. Wornell, "Cooperative diversity in wireless networks: Efficient protocols and outage behavior," IEEE Trans. Inf. Theory, vol. 50, no. 11, pp. 3062-3080, Nov. 2004.

[5] O. Simeone, Y. Bar-Ness, and U. Spagnolini, "Stable throughput of cognitive radios with and without relaying capability," IEEE Trans. Commun., vol. 55, no. 12, pp. 2351-2360, Dec. 2007.

[6] Q. Zhang, J. ia, and J. Zhang, "Cooperative relay to improve diversity in cognitive radio networks," IEEE Commun. Mag., vol. 47, no. 2, pp. 111-117, Feb. 2009.

[7] H. Su and X. Zhang, "Cross-layer based opportunistic MAC protocols for QoS provisionings over cognitive radio mobile wireless networks," IEEE J. Sel. Areas Commun., vol. 26, no. 1, pp. 118-129, Jan. 2008.

[8] T. Cover and A. Gamal, "Capacity theorems for the relay channel," IEEE Trans. Inf. Theory, vol. 25, no. 5, pp. 572-584, Sept. 1979.

[9] M. Khojastepour, A. Sabharwal, and B. Aazhang, "On capacity of Gaussian 'cheap' relay channel," in IEEE GLOBECOM'03, San Francisco, CA, Dec. 2003, pp. 1776-1780.

[10] Y. Zhao, R. Adve, and T. Lim, "Improving amplify-and-forward relay networks: Optimal power allocation versus selection," IEEE Trans. Wireless Commun., vol. 6, no. 8, pp. 3114-3123, Aug. 2007.

[11] A. Bletsas, A. Khisti, D. Reed, and A. Lippman, "A simple cooperative diversity method based on network path selection," IEEE J. Sel. Areas Commun., vol. 24, no. 3, pp. 659-672, Mar. 2006.

[12] T. C.-Y. Ng and W. Yu, "Joint optimization of relay strategies and resource allocations in cooperative cellular networks," IEEE J. Sel. Areas Commun., vol. 25, no. 2, pp. 328-339, Feb. 2007.

[13] J. Cai, X. Shen, J. Mark, and A. Alfa, "Semi-distributed user relaying algorithm for amplify-and-forward wireless relay networks," IEEE Trans. Wireless Commun., vol. 7, no. 4, pp. 1348-1357, Apr. 2008.

[14] Y. Shi, S. Sharma, Y. Hou, and S. Kompella, "Optimal relay assignment for cooperative communications," in ACM MobiHoc'08, Hong Kong, China, May 2008, pp. 3-12.

[15] L. Ding, T. Melodia, S. Batalama, and J. Matyjas, "Distributed routing, relay selection, and spectrum allocation in cognitive and cooperative ad hoc networks," in Proc. IEEE SECON'10, Boston, MA, June 2010. 